\section{Journal Club}

der Gruppe mit den Raffungsoperationen auf $(5, .8 \%$ versus $3,8 \%)$.

Diskussion. Gemäß der Datenlage war kein signifikanter Unterschied in den Langzeitergebnissen zwischen den beiden Verfahren feststellbar. Bekannt ist jedoch eine wesentlich höhere 30-TageMortalität der Klappenersatzoperation mit 8,5\% versus 5,3\% bei der Raffungsoperation. Allerdings war die Rate an Rezidiven mit Mitralinsuffizienz und daraus resultierender Herzinsuffizienz in der Gruppe mit Raffungsoperationen höher. Die Indikationsstellung wird gemäß diesen Daten wieder in Richtung Mitralklappenersatzoperation gehen.

Prim. Dr. Peter Dovjak

\section{Korrespondenzadresse}

\section{PD Dr. R. Püllen}

Agaplesion Frankfurter Diakonie-Kliniken Holzhausenstr. 72-92, 60322 Frankfurt am

Main, Deutschland

rupert.puellen@fdk.info

\section{Prim. Dr. P. Dovjak}

Salzkammergut-Klinikum

Miller-von-Aichholz-Str. 49, 4810 Gmunden, Österreich

peter.dovjak@gespag.at

Interessenkonflikt. R. Püllen, P. Dovjak, C. Gisinger und H. Burkhardt geben an, dass kein Interessenkonflikt besteht.

\title{
Erratum
}

Z Gerontol Geriat 2016 · 49:263-264

DOI 10.1007/s00391-016-1059-4

Online publiziert: 8. April 2016

(c) Springer-Verlag Berlin Heidelberg 2016

CrossMark

\section{P. Dovjak' $\cdot$ R. Püllen}

'Salzkammergut-Klinikum, Gmunden, Österreich

${ }^{2}$ Agaplesion Frankfurter Diakonie-Kliniken, Frankfurt am Main, Deutschland

\section{Erratum zu: Journal Club}

\section{Verbessert die intensivierte Senkung des Blutdrucks (systolisch 120 mmHg als Zielwert) bei Patienten mit arterieller Hypertonie die Mortalitåt?}

\author{
Erratum zu: \\ Z Gerontol Geriat (2016) \\ 49:166-167 \\ DOI:10.1007/s00391-015-1017-6
}

Dieser Beitrag wurde versehentlich einem falschen Autor zugeordnet. Der richtige Autor lautet:

PD Dr. med. H. Burkhardt, Mannheim

Die Redaktion bittet, diesen Fehler zu entschuldigen.

\section{Korrespondenzadresse}

\section{Dr. P. Dovjak}

Salzkammergut-Klinikum 4810 Gmunden, Österreich peter.dovjak@gespag.at

\section{PD Dr. R. Püllen}

Agaplesion Frankfurter Diakonie-Kliniken Holzhausenstr. 72-92, 60322 Frankfurt am Main, Deutschland rupert.puellen@fdk.info
Die Online-Version des Originalartikels ist unter http://dx.doi.org/10.1007/s00391-015-1017-6 zufinden. 
Hier steht eine Anzeige.

算 Springer 